\title{
Deformable mirror fitting error by correcting the segmented wavefronts
}

\author{
Natalia Yaitskova ${ }^{a}$ \\ European Organization for Astronomical Research in the Southern Hemisphere (ESO) \\ Karl Schwarzschildstr. 2, D-85748 Garching b. Muenchen, Germany
}

\begin{abstract}
The objective of the present study is to estimate the ability of conventional adaptive optics $(\mathrm{AO})$ to correct for a wavefront created by static errors randomly distributed over the population of a multi-segmented primary mirror for an extremely large telescope (ELT). Among all errors the deformable mirror fitting error is the factor which contributes the most to the performance degradation. We quantify this error through a ratio between two root mean square (RMS) values of the phase before and after the correction. We compare the results of several AO models: from the high-pass hard edge filter to the optical waves end-to-end modeling.
\end{abstract}

\section{Introduction}

In general, the wavefront control of Extremely Large Telescope (ELT) includes three main units: adaptive optics (AO), active optics and phasing camera. The corresponding control loops run independently. Each unit is meant to correct for the different components of the distorted wavefront. Disentangling of the components is based on their difference in temporal and spatial bandwidths. Nevertheless, each wavefront control unit is affected to some extend by the total wavefront. Our objective is to quantify the ability of adaptive optics to compensate for the part of the distorted wavefront produced by segmentation of the primary mirror. The discontinuities are due to the aberrations of the individual segments. The aberrations might be residuals of the phasing system (piston, tip and tilt), the segment shape control with the warping harnesses and/or the residuals of segment polishing. The errors between the different segments are assumed to be uncorrelated, which represents a worst-case scenario for the imaging. The adaptive optics of either the telescope or in the instrument (or both) sees these distortions and tries to compensate for them by adjusting the shape of the deformable mirror (DM). Naturally, the more degrees of freedom adaptive optics has, the better the possible correction. The level of correction is evaluated through the RMS improvement criterion: the ratio between the RMS values of corrected and uncorrected wavefronts. First we introduce the AO in a very abstract way: modeled as a hard-edge filter of the phase. Later on we argue that the hard-edge filter correction level can never be achieved in practice. We claim that the realistic RMS improvement will always be lower than the one from the hard-edge filter model. Putting together the results from the different AO models, including the least-square fitting, we estimate the expected correction in term of RMS in optimistic and pessimistic cases, introducing definitions for "good" and "medium" quality AO correspondingly.

\section{Ultimate Performance of Adaptive Optics}

We consider a segmented telescope with all identical segments of a given shape. The phase error on each individual segment can be represented as a set of aberrations, described for example by a Zernike polynomial. We use the term segment aberration to refer to a particular aberration of an individual segment and the term segmentation error to refer to the shape

\footnotetext{
a e-mail: nyaitsko@eso.org
}

This is an Open Access article distributed under the terms of the Creative Commons Attribution-Noncommercial License, which permits unrestricted use, distribution, and reproduction in any noncommercial medium, provided the original work is properly cited. 
of the wavefront reflected by the whole segmented mirror. At first we do not specify a type of aberration; it allows us to present a model of diffraction in a general way. In this paper, we study the effect of different aberrations separately; therefore the segmentation error is described by only one polynomial $Z_{n}$ with the coefficients $a_{j n}$ randomly distributed over the whole population of the segments:

$$
\phi(\mathbf{x})=\sum_{j=1}^{N} a_{j n} \theta\left(\mathbf{x}-\mathbf{r}_{j}\right) Z_{n}\left(\mathbf{x}-\mathbf{r}_{j}\right) .
$$

In this equation $\mathbf{r}_{j}$ describes the central position of the segment with index $j$ and $\theta(\mathbf{x})$ is the segment transmission function, which takes 1 inside the segment area and 0 outside it. For the following analysis we will need an expression for the initial PSD of the segmentation error given by Eq.(1). In can be written in a nice compact form [1] provided that the coefficients $a_{j n}$ are uncorrelated:

$$
\begin{aligned}
P S D_{\text {ini }}(\mathbf{w}) & =\left(\frac{r m s_{\text {ini }}}{\tau_{n}}\right)^{2} \frac{1}{N} \mid(1 / A) \int Z_{n}(\xi) \theta(\xi) \exp \left[\left.(i(2 \pi / \lambda) \mathbf{w} \xi) d^{2} \xi\right|^{2}\right. \\
& =\left(\frac{r m s_{\text {ini }}}{\tau_{n}}\right)^{2} \frac{1}{N}\left|t_{n}(\mathbf{w})\right|^{2}
\end{aligned}
$$

where $r m s_{\text {ini }}$ is the initial RMS of the segmentation error ( $n$ is the index of the aberration), $\tau_{n}$ is a form-factor: a rational number appearing while using Zernike polynomials within a hexagonal area; $A$ is the total area of a hexagon. The Fourier transform of the considered aberration in a hexagonal area can be calculated analytically [1],[2]. Considering the first, most simple model of the $\mathrm{AO}$ we assume that the phase correction is analogous to the modification or filtering of the phase power spectral density (PSD). The shape of the filter depends on the particular design of the AO system and included errors. The area where the PSD is modified is called the control region $\left(G_{A O}\right)$ and is limited by the deformable mirror cut-off frequency. An ideal AO system completely cancels the PSD inside $G_{A O}$. This type of AO is usually simulated as a top-head spatial filter applied on the initial phase. According to the Parseval's theorem the RMS is related to the PSD by a simple integral relation. Therefore, for the hard-edge filter model, the final RMS value is:

$$
r m s_{\text {fin }, u l t}^{2}=\frac{A N}{\lambda^{2}} \int_{G_{A O}^{C}} P S D_{i n i}(\mathbf{w}) d^{2} w
$$

where $G_{A O}^{C}$ is a complement of $G_{A O}$, i.e the integral is taken outside the control region. From Eq.(2) and Eq.(3) we conclude that a fundamental parameter of this model of AO is a ratio between the final and the initial RMS values. According to the adopted notation [3], we call this ratio a correction factor, $\gamma_{A O}$ :

$$
\gamma_{A O}=\frac{r m s_{f i n}}{r m s_{\text {ini }}}
$$

This value is independent of the initial RMS, the number of segments and the wavelength. To calculate the best correction factor one needs to know only two inputs: the type of aberration and the geometry of the control region. Suppose that the control region is rectangular and bounded by a cut-off frequency of the DM: $\pm \lambda / 2 d_{a}$, where $d_{a}$ is the distance between the virtual actuators projected onto the pupil plane. In the normalized coordinates ( $\alpha=\pi d w_{x} / 2 \lambda, \beta=\pi d w_{y} / 2 \lambda, d$ is flat to flat segment size) the cut-off frequency is $\pi / 4\left(d / d_{a}\right)$. Thus, the main parameter is the ratio between the segment size and the inter-actuator separation, $m=d / d_{a}$. The best correction factor in the case of hexagonal segments and a rectangular control region:

$$
\gamma_{A O, u l t}=\left[1-\frac{2 \sqrt{3}}{\pi^{2} \tau_{n}} \int_{-\frac{\pi m}{4}}^{\frac{\pi m}{4}} d \alpha \int_{-\frac{\pi m}{4}}^{\frac{\pi m}{4}} d \beta\left|t_{n}(\alpha, \beta)\right|^{2}\right]^{1 / 2}
$$




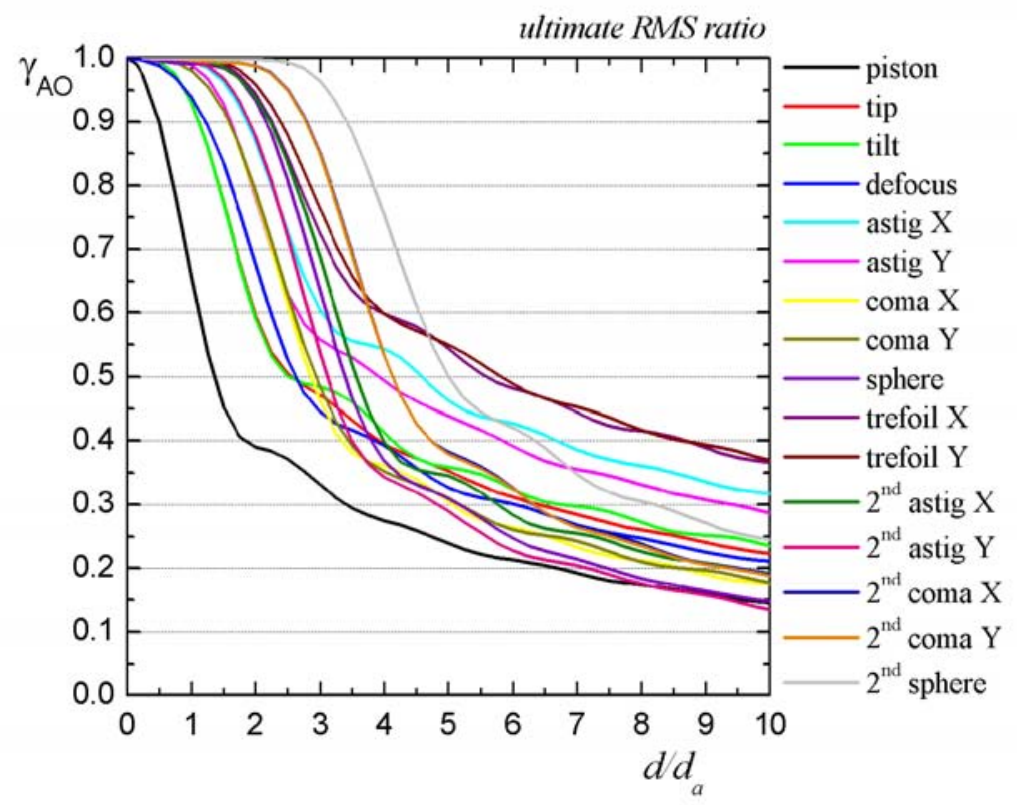

Fig. 1. Ultimate correction factor for different aberrations as a function of a segment size related to an actuator pitch

The lower the correction factor, the better the performance of the AO. The improvement factor can be estimated as $1-\gamma_{A O}$. The best correction factor for the first 16 Zernike polynomials is shown in Figure 1 as a function of the actuators' density. As one can see, the aberration order in terms of RMS improvement is not the same for the low order AO $(m \sim 2)$ and the extreme AO $(m \sim 6)$. Aberrations can be split into groups by the order of RMS improvement. Piston is the best correction aberration for any regime. For low order AO the groups are: piston (60\% improvement); tip and tilt (40\% improvement); defocus (30\% improvement); astigmatism Y, coma X and coma Y (20\% improvement); astigmatism X, 2nd astigmatism $\mathrm{Y}$ (12\% improvement); spherical aberration, 2nd astigmatism X, trefoil X and trefoil $\mathrm{Y}$, 2nd coma $\mathrm{X}$ and 2nd coma $\mathrm{Y}$, and second spherical aberration are almost uncorrected $(1-5 \%)$. In case of extreme $\mathrm{AO}$ the order changes. We can conventionally select the groups of aberrations: piston, 2nd astigmatism Y, spherical aberration (80\% improvement); coma X, coma Y ( $76 \%$ improvement); 2nd astigmatism X, 2nd coma X, 2nd coma Y, defocus (74\% improvement); tip and tilt (72\% improvement); astigmatism X, astigmatism Y, second spherical aberration (640\% improvement); trefoil X, trefoil Y (55\% improvement). The discrepancy between X and $\mathrm{Y}$ components of the same aberration are explained by the fact that for the hexagonal geometry, $\mathrm{X}$ and $\mathrm{Y}$ directions are not equivalent: rotation of a segment by $\pi / 2$ does not transfer one component of the aberration into another.

\section{More realistic models of adaptive optics}

Real AO includes many sources of error [4]: DM fitting, spatial aliasing, servo-lag, photon noise and detector noise, anisoplanatic error. Due to this, the residual RMS is higher than the one in our best case. For us it means that the PSF of the residual phase will have a remaining second order halo inside the control region. For the segmentation error, temporal errors are negligible: there is no delay between the measurement and correction for the quasi-static wavefront. For the same reason the photon noise is not an issue: long exposures can be used to increase the signal to noise ratio. The problem of anisoplanatism does not arise here because the physical 
source of the wavefront distortions is located in one plane only. Therefore the main source of residual AO error for the segment aberrations is DM fitting. The fitting error arises when a continuous DM with given properties (actuator spacing and geometry, influence function shape, coupling, etc.) reconstructs a wavefront.

There exist three principal models of DM action on the wavefront: modal control, when the low-order aberrations of the whole mirror are completely corrected; the filtering approach, when the DM influence is described as a low pass filter on the phase [5]; and a zonal model, when the DM is locally deformed by displacements of the actuators.

The modal control neglects the effect of the pupil border. It is applicable to the monolithic mirror case, where the border is outside of the imaging pupil. It is not applicable to the case of segmentation, where the pupil parts containing the segment borders play a role in image construction and hence affect the image quality. The modal approach therefore does not fit our task.

The filtering approach is barely applicable here either. The spectral corrective ability of adaptive optics cannot be described in terms of a transfer function. The actuators exist in specific locations and the mirror does not respond in a shift invariant fashion. This is shown in [6]. The argument is as follows: imagine a wavefront shifted laterally in the optical plane. The shifting has no effect on the spectrum of the incident wavefront. However, the least-square fit of the mirror surface will not be the same for the shifted and un-shifted wavefronts due to the inability of the mirror to match the shift. The reflected spectrum is different in each case, so the transfer function is not unique.

If one can use the filtering approach as an approximation for a homogeneous wavefront such as a turbulent atmosphere, for an inhomogeneous wavefront (such as segmentation error) the situation is even more critical. In this case not only is the DM not invariant by the translation, but the wavefront is as well. The lateral shift changes the geometrical properties of the wavefront, unless this shift is equal to an integer number of segment sizes. Nevertheless, we will now assume that a realistic AO system preserves the linear property of the ideal one: suppose that the correction gain does not depend on the initial value of the error, and that the performance can be quantified by the correction factor. What is the value of the correction factor for a realistic AO and how it is related to the best case?

We start with the least-square fit model. The DM response is composed of influence functions with pre-defined profiles. We assume that the influence function for each actuator is the same. To avoid an ambiguity, we allow no actuators to be placed between the segments. To derive the general expressions independent of a particular mirror configuration the fitting procedure is applied to each segment separately. This approach allows us to avoid performing simulations of the segmentation error and to use a quasi-analytical approach. The drawback is that in this method the fitting of phase steps is not optimized.

Let $I F\left(\xi-\xi_{l}\right)$ be an influence function centered at the position of an actuator $\xi_{l}$ in the system of coordinates bounded with the center of the segment. We have to find a set of coefficients $\kappa_{n l}$, describing the actuators' response to an aberration $Z_{n}$ of unit value, which are minimizing the residual RMS value. If we sample the segment area $\xi=\xi_{i}$ and introduce a matrix $H_{i l}=I F\left(\xi_{i}-\xi_{l}\right)$ and a vector $B_{i}=Z_{n}\left(\xi_{i}\right) \theta\left(\xi_{i}\right)$, then the process of minimizing RMS is analogous to the minimizing a norm of the vector $H \kappa-B$. The solution is well-known least-square fit with the residual error:

$$
e^{2}=B\left[I-H\left(H^{t} H\right)^{-1} H^{t}\right] B^{t}
$$

where $I$ is the unitary matrix and $t$ means matrix transpose. The residual error depends on the distribution of the actuators over the segment. If the configuration is the same for each segment, the RMS improving factor in this model is

$$
\gamma_{A O, L S}=\sqrt{\frac{e^{2}}{\tau_{n} \tilde{A}}}
$$


Natalia Yaitskova: Deformable mirror fitting error by correcting the segmented wavefronts
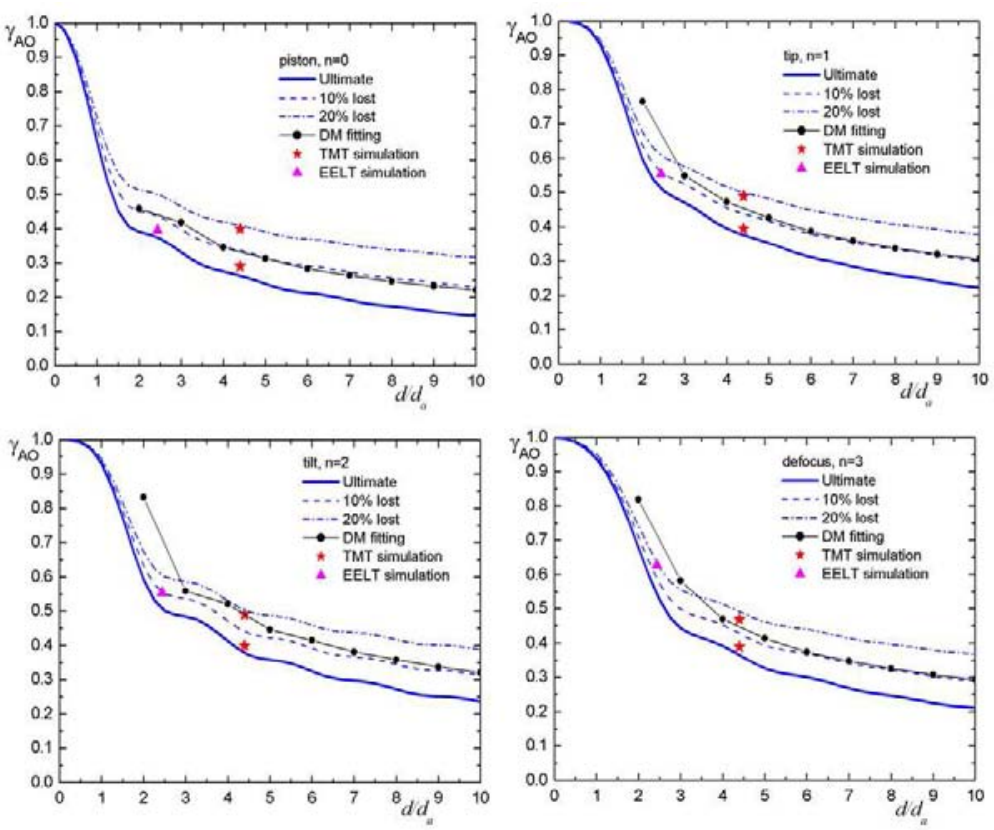

Fig. 2. Correction factor for different AO models for piston. tip, tilt and defocus

where $\tilde{A}$ is a number of sampling points covering the segment transmission function. If the configuration of the actuators changes from segment to segment, the $\gamma_{A O, L S}$ must be calculated as a square average. We have performed the calculation for hexagonal segments, Zernike polynomials and the cubic spline influence function with $25 \%$ coupling. We use four actuators configurations for the averaging. Sampling is $300 \times 300$ points on a square $1.7 d$ by $1.7 d ; \tilde{A}=$ 25959. The results are presented in Figure 2 (circles).

As we already mentioned, the algorithm is not optimal when each segment is fitted separately. We also performed simulations fitting the whole phase map representing a particular realization of segmentation error. To distinguish it from the method above we call it global fitting. To simulate the segmentation error, we use the model of the E-ELT primary mirror with 984 segments and populate the aberration randomly over the segments (central normal distribution). The size of each segment is $d=1.22 \mathrm{~m}$. The model of the DM corresponds to the deformable mirror embedded into the E-ELT optics with actuator separation of $50 \mathrm{~cm}$ (projected onto primary mirror): $m=2.44$.

The correction factor is included in Figure 2 (triangles). This algorithm yields much better results than fitting an individual segment. For the low order AO, separate segment to segment fitting gives an unrealistic estimation and shouldn't be used. Although we do not have at our disposal results for global fitting with a higher density of actuators, we expect that for the extreme AO case two ways of fitting will produce similar results.

On each plot of Figure 2 the thick line is the best correction factor, circles represent the one obtained from least square fitting when the aberration on each segment is fitted separately. Triangles are the results of the global fitting simulations. Stars are the results of the TMT simulation taken from the work by Crossfield and Troy [7], the upper star is the result of the wave-optics simulation using the Arroyo library. They used a Shack-Hartmann model with an $127 \times 127$ actuator deformable mirror and a spatial filtered Shack-Hartmann wavefront sensor acting on the unobscured TMT aperture $(m=4.4)$. The lower star is the result from the same paper obtained with a hard-edge high-pass filter. By definition, these results must coincide with our best correction factor. We took care on the swapping the $\mathrm{X}$ and $\mathrm{Y}$ components for the aberrations when placing the TMT results on our plots. 


\section{4 "Good" and "medium" quality adaptive optics}

One of the main applications of this study is establishing the requirements of the segmentation error for an ELT. As we cannot predict the AO technology of the future, for technology- and model-free estimation we introduce the concept of "good" and "medium" quality AO. The "good" quality AO addresses the optimistic assumption of $10 \%$ loss in performance with respect to our best case. The "medium" quality AO addresses the pessimistic assumption of $20 \%$ performance loss. The corresponding RMS improvement is

$$
\begin{gathered}
1-\gamma_{A O, \text { good }}=0.9\left(1-\gamma_{A O, u l t}\right), \\
1-\gamma_{A O, \text { medium }}=0.8\left(1-\gamma_{A O, u l t}\right) .
\end{gathered}
$$

On the graphs in Figure 2 we plot curves corresponding to the correction factor for the two cases. Notice that for the extreme AO case the results of a least-square segment by segment fitting fall into the "optimistic" category. The results of the wave-optics simulation are either $10 \%$ or $20 \%$ depending on the type of aberration, as well as the results of the global fitting. As segment by segment fitting, global fitting and the TMT simulations were performed with completely independent AO modes (and by different people), we are confident that the 10-20\% range covers most cases.

The difference between the correction factor of the realistic AO and our best correction factor defines the integral value of the residual PSD within the control region. From the relation connecting the PSD and RMS we can express the mean level of the halo inside the control area through the initial RMS and the correcting factor.

$$
\overline{P S D}_{f i n}=\left[\gamma_{A O}^{2}-\gamma_{A O, u l t}^{2}\right] \frac{r m s_{i n i}^{2}}{N} \frac{2}{\sqrt{3}} \frac{1}{m^{2}} .
$$

One can use this equation either to estimate the level of the residual halo withing a control region by given initial RMS, or, inversely, to establish the level of the initial RMS which provides the residual PSD with the required mean level. As an example, we have chosen the application of high contrast imaging. The distorted wavefront containing the star and the planet after entering the instrument is corrected by the extreme AO. The actuator pitch in the extreme AO case is about $20 \mathrm{~cm}$. For a segment size of $1.22 \mathrm{~m}$ we obtain: $m=6$. We define the requirements on the segment aberration by fixing the level of the residual halo after the extreme AO module. This component of the wavefront error is not altered by a coronagraph (if coronagraph does not belong to interferometric type). We ask the level of the residual halo to be equal to the level of the residual diffraction delivered by a coronagraph. This level is typically $10^{-7}$ times the peak intensity of the initial non-aberrated image. Each aberration shall introduce no more than $10 \%$ of this level, i.e. the halo level from each separate aberration shall be $10^{-8}$. With increasing aberration order, the initial level of the PSD lowers, but the correction ability lowers as well, and hence the required level of the initial RMS for different aberrations is similar. Solving Eq.(9) with $\overline{P S D}_{\text {fin }}=10^{-8}, N=984$ and $m=6$ we obtained $r m s_{i n i}=20 \mathrm{~nm}$ for "good" quality $\mathrm{AO}$, and $r m s_{i n i}=14 \mathrm{~nm}$ for "medium" quality AO in the H-band $(1.6 \mu m)$.

\section{References}

1. N. Yaitskova, JOSAA 26, (2009) 59-71

2. N. Yaitskova, proc. SPIE 7012, (2008) 40

3. N. Yaitskova and C. Verinaud proc. SPIE 6267, (2006) 75

4. F. Rigaut, J.-P. Veran and O. Lai, proc. SPIE 3353,(1998) 1038-1048

5. J. E. Harvey and G. M. Callahan, Proc. Soc. Photo-Opt.Instrum. Eng. 141, (1978) 50-57

6. K. E. Moor and G. N. Lawrence, Appl. Opt. 29, (1990) 4622-4628

7. J. Crossfield and M. Troy, Appl. Opt. 46, (2007) 4533-4540 